\title{
Approximation by a generalized Szász type operator for functions of two variables
}

Nursel Çetin, Sevilay Kirci Serenbay, and Çĭgdem Atakut 


\title{
APPROXIMATION BY A GENERALIZED SZÁSZ TYPE OPERATOR FOR FUNCTIONS OF TWO VARIABLES
}

\author{
NURSEL ÇETIN, SEVILAY KIRCI SERENBAY, AND ÇIĞDEM ATAKUT \\ Received 05 December, 2012
}

\begin{abstract}
In the present paper, we define a new Szász-Mirakjan type operator in exponential weighted spaces for functions of two variables having exponential growth at infinity using a method given by Jakimovski-Leviatan. This operator is a generalization of two variables of an operator defined by A. Ciupa [1]. In this study, we investigate approximation properties and also estimate the rate of convergence for this new operator.
\end{abstract}

2010 Mathematics Subject Classification: 41A25;41A36

Keywords: linear positive operator, Jakimovski-Leviatan operator, weighted space, modulus of continuity, rate of convergence

\section{INTRODUCTION}

For a real function of real variable $f:[0, \infty) \rightarrow \mathbb{R}$, the Szász-Mirakjan operators are defined in [2] as

$$
S_{n}(f ; x)=e^{-n x} \sum_{j=0}^{\infty} \frac{(n x)^{j}}{j !} f\left(\frac{j}{n}\right), x \in[0, \infty),
$$

where the convergence of $S_{n}(f ; x)$ to $f(x)$ under the exponential growth condition on $f$ that is $|f(x)| \leq C e^{B x}$, for all $x \in[0, \infty)$, with $C, B>0$ was proved. Then, various modifications and further properties of the Szász-Mirakjan operators have been studied intensively by many authors (e.g. [1,3-9]).

In [4], A. Jakimovski and D. Leviatan investigated approximation properties of a generalization of the Szász-Mirakjan operators which are stated as follows:

Let $g(z)=\sum_{n=0}^{\infty} a_{n} z^{n}$ be an analytic function in the disk $|z|<R, R>1$ and suppose $g(1) \neq 0$. Define the Appell polynomials $p_{k}(x)=p_{k}(x, g)(k \geq 0)$ by

$$
g(u) e^{u x}=\sum_{k=0}^{\infty} p_{k}(x) u^{k}
$$


For each function $f$ defined in $[0, \infty)$, they considered the operators $L_{n}$ defined by

$$
L_{n}(f ; x)=\frac{e^{-n x}}{g(1)} \sum_{k=0}^{\infty} p_{k}(n x) f\left(\frac{k}{n}\right), n>0
$$

and also the authors obtained several approximation properties of these operators. A. Ciupa [1] introduced a Szász-Mirakjan type operator that is a generalization of the operator defined by M. Lesniewicz and L. Rempulska [5] using the method given by Jakimovski-Leviatan. A. Ciupa studied the properties of approximation for functions of one variable in the space of continuous functions having an exponential growth at infinity.

In this paper, inspired by [1], for each function $f$ defined in $[0, \infty) \times[0, \infty)$, we define the operators $L_{n, m}$ by

$$
L_{n, m}(f ; x, y)=\frac{e^{-n x} e^{-m y}}{(g(1))^{2}} \sum_{k=0}^{\infty} \sum_{j=0}^{\infty} p_{k}(n x) p_{j}(m y) f\left(\frac{k}{n}, \frac{j}{m}\right)
$$

where

$$
g\left(u_{1}\right) e^{u_{1} x} g\left(u_{2}\right) e^{u_{2} y}=\sum_{k=0}^{\infty} p_{k}(x) u_{1}^{k} \sum_{j=0}^{\infty} p_{j}(y) u_{2}^{j} .
$$

Now, we consider the function $g(x)=\sum_{k=0}^{\infty} \frac{x^{2 k+1}}{(2 k+1) !}=\sinh x$ where $\sinh x$ is the hyperbolic function of $x$ and let $p_{k}$ be the polynomials generated by relation

$$
\sinh u_{1} \sinh \left(u_{1} x\right) \sinh u_{2} \sinh \left(u_{2} y\right)=\sum_{k=0}^{\infty} \sum_{j=0}^{\infty} p_{2 k}(x) p_{2 j}(y) u_{1}^{2 k} u_{2}^{2 j} .
$$

Using the following equalities

$$
\begin{aligned}
& \sinh u_{1} \sinh \left(u_{1} x\right)=\frac{1}{2} \sum_{k=0}^{\infty} \frac{(1+x)^{2 k}-(1-x)^{2 k}}{(2 k) !} u_{1}^{2 k} \\
& \sinh u_{2} \sinh \left(u_{2} y\right)=\frac{1}{2} \sum_{j=0}^{\infty} \frac{(1+y)^{2 j}-(1-y)^{2 j}}{(2 j) !} u_{2}^{2 j},
\end{aligned}
$$

we have

$$
p_{2 k}(x)=\frac{(1+x)^{2 k}-(1-x)^{2 k}}{2(2 k) !}, \quad p_{2 j}(y)=\frac{(1+y)^{2 j}-(1-y)^{2 j}}{2(2 j) !} .
$$

Let $C\left(R_{1}^{2}\right)$ be the set of all real-valued continuous functions of two variables on $R_{1}^{2}:=\{(x, y): x \geq 1, y \geq 1\}$. 
For $p, q>0$ and $(x, y) \in R_{1}^{2}$, we define

$$
\begin{aligned}
w_{p, q}(x, y) & =w_{p}(x) w_{q}(y)=e^{-p x} e^{-q y} \\
C_{p, q} & =\left\{f \in C\left(R_{1}^{2}\right): w_{p, q} f \text { is uniformly continuous and bounded on } R_{1}^{2}\right\} \\
\|f\|_{p, q} & =\sup _{(x, y) \in R_{1}^{2}} w_{p}(x) w_{q}(y)|f(x, y)|
\end{aligned}
$$

and also for $h, k \geq 0, \delta \geq 0, f \in C_{p, q}$, the first order modulus of continuity given by

$$
\omega\left(f, C_{p, q} ; \delta\right)=\sup _{0 \leq h, k \leq \delta}\left\|\Delta_{h, k} f\right\|_{p, q}
$$

where

$$
\Delta_{h, k} f(x, y)=f(x+h, y+k)-f(x, y) .
$$

In this study, in the space $C_{p, q}, p, q>0$, we introduce the following positive linear operators

$$
P_{n, m}(f ; x, y)=\frac{1}{(\sinh 1)^{2} \sinh (n x) \sinh (m y)} \sum_{k=0}^{\infty} \sum_{j=0}^{\infty} p_{2 k}(n x) p_{2 j}(m y) f\left(\frac{2 k}{n}, \frac{2 j}{m}\right)
$$

$n, m \in \mathbb{N},(x, y) \in R_{1}^{2}$ and investigate the theorems on convergence of $P_{n, m}(f ; x, y)$ operators to functions of two variables. We also estimate the rate of convergence for this new operator by using the modulus of continuity.

\section{Auxiliary Results}

In this section, we will give some useful results in order to study the convergence of the sequence $\left(P_{n, m} f\right)$ to the function $f \in C_{p, q}$.

Lemma 1. If $(x, y) \in R_{1}^{2}$ and $n, m \in N$, we have

$$
\begin{aligned}
P_{n, m}\left(e_{0,0} ; x, y\right) & =1 \\
P_{n, m}\left(e_{1,0} ; x, y\right) & =\frac{1}{n} \operatorname{coth} 1+x \operatorname{coth}(n x) \\
P_{n, m}\left(e_{0,1} ; x, y\right) & =\frac{1}{m} \operatorname{coth} 1+y \operatorname{coth}(m y) \\
P_{n, m}\left(e_{1,0}^{2}+e_{0,1}^{2} ; x, y\right)= & \left(x^{2}+y^{2}\right)+\left(\frac{1}{n^{2}}+\frac{1}{m^{2}}\right)(1+\operatorname{coth} 1) \\
& +(1+2 \operatorname{coth} 1)\left(\frac{x}{n} \operatorname{coth}(n x)+\frac{y}{m} \operatorname{coth}(m y)\right)
\end{aligned}
$$

where $e_{i, j}\left(t_{1}, t_{2}\right)=t_{1}^{i} t_{2}^{j} ; i, j \in\{0,1\}$ and $\operatorname{coth} u$ is the hyperbolic function of $u$. 
Lemma 2. If $(x, y) \in R_{1}^{2}, p, q>0$ and $n, m \in N$, then we have

$$
\begin{gathered}
P_{n, m}\left(e^{p t_{1}} ; x, y\right)=\frac{1}{\sinh 1 \sinh (n x)} \sinh \left(e^{p / n}\right) \sinh \left(n x e^{p / n}\right) \\
P_{n, m}\left(e^{q t_{2}} ; x, y\right)=\frac{1}{\sinh 1 \sinh (m y)} \sinh \left(e^{q / m}\right) \sinh \left(m y e^{q / m}\right) \\
P_{n, m}\left(t_{1} e^{p t_{1}} ; x, y\right)=\frac{e^{p / n}}{n} \frac{1}{\sinh 1 \sinh (n x)}\left\{\cosh \left(e^{p / n}\right) \sinh \left(n x e^{p / n}\right)\right. \\
\left.+n x \sinh \left(e^{p / n}\right) \cosh \left(n x e^{p / n}\right)\right\} \\
P_{n, m}\left(t_{2} e^{q t_{2}} ; x, y\right)=\frac{e^{q / m}}{m} \frac{1}{\sinh 1 \sinh (m y)}\left\{\cosh \left(e^{q / m}\right) \sinh \left(m y e^{q / m}\right)\right. \\
P_{n, m}\left(t_{1}^{2} e^{p t_{1}} ; x, y\right)=\frac{1}{\sinh 1 \sinh (n x)}\left\{\frac{e^{2 p / n}}{n^{2}} \sinh \left(e^{p / n}\right) \sinh \left(n x e^{p / n}\right)\right. \\
+\frac{2 x}{n} e^{2 p / n} \cosh \left(e^{p / n}\right) \cosh \left(n x e^{p / n}\right)+x^{2} e^{2 p / n} \sinh \left(e^{p / n}\right) \sinh \left(n x e^{p / n}\right) \\
\left.+\frac{1}{n^{2}} e^{p / n} \cosh \left(e^{p / n}\right) \sinh \left(n x e^{p / n}\right)+\frac{x}{n} e^{p / n} \sinh \left(e^{p / n}\right) \cosh \left(n x e^{p / n}\right)\right\} \\
P_{n, m}\left(t_{2}^{2} e^{q t_{2}} ; x, y\right)=\frac{1}{\sinh 1 \sinh (m y)}\left\{\frac{e^{2 q / m}}{m^{2}} \sinh \left(e^{q / m}\right) \sinh \left(m y e^{q / m}\right)\right. \\
+\frac{2 y}{m} e^{2 q / m} \cosh \left(e^{q / m}\right) \cosh \left(m y e^{q / m}\right)+y^{2} e^{2 q / m} \sinh \left(e^{q / m}\right) \sinh \left(m y e^{q / m}\right) \\
\left.+\frac{1}{m^{2}} e^{q / m} \cosh \left(e^{q / m}\right) \sinh \left(m y e^{q / m}\right)+\frac{y}{m} e^{q / m} \sinh \left(e^{q / m}\right) \cosh \left(m y e^{q / m}\right)\right\}
\end{gathered}
$$

Lemma 3. For all $(x, y) \in R_{1}^{2}$ and $n, m \in N$, we have

$$
\begin{aligned}
& P_{n, m}\left(\left(t_{1}-x\right)^{2} e^{p t_{1}} ; x, y\right) \\
& =\frac{1}{\sinh 1 \sinh n x}\left\{x^{2} \sinh \left(e^{p / n}\right) \sinh \left(n x e^{p / n}\right)\left[e^{p / n}-1\right]^{2}\right. \\
& \quad+\sinh \left(n x e^{p / n}\right)\left[\frac{e^{2 p / n}}{n^{2}} \sinh \left(e^{p / n}\right)+\frac{e^{p / n}}{n^{2}} \cosh \left(e^{p / n}\right)\right. \\
& \left.\quad-\frac{2 x}{n} e^{p / n} \cosh \left(e^{p / n}\right)\right]+\cosh \left(n x e^{p / n}\right)\left[\frac{2 x}{n} e^{2 p / n} \cosh \left(e^{p / n}\right)\right.
\end{aligned}
$$




$$
\begin{aligned}
& \left.\left.\quad+\frac{x}{n} e^{p / n} \sinh \left(e^{p / n}\right)\right]-2 x^{2} e^{p / n} \sinh \left(e^{p / n}\right) e^{-n x e^{p / n}}\right\} \\
& P_{n, m}\left(\left(t_{2}-y\right)^{2} e^{q t_{2}} ; x, y\right) \\
& =\frac{1}{\sinh 1 \sinh m y}\left\{y^{2} \sinh \left(e^{q / m}\right) \sinh \left(m y e^{q / m}\right)\left[e^{q / m}-1\right]^{2}\right. \\
& \quad+\sinh \left(m y e^{q / m}\right)\left[\frac{e^{2 q / m}}{m^{2}} \sinh \left(e^{q / m}\right)+\frac{e^{q / m}}{m^{2}} \cosh \left(e^{q / m}\right)\right. \\
& \left.\quad-\frac{2 y}{m} e^{q / m} \cosh \left(e^{q / m}\right)\right]+\cosh \left(m y e^{q / m}\right)\left[\frac{2 y}{m} e^{2 q / m} \cosh \left(e^{q / m}\right)\right. \\
& \left.\left.\quad+\frac{y}{m} e^{q / m} \sinh \left(e^{q / m}\right)\right]-2 y^{2} e^{q / m} \sinh \left(e^{q / m}\right) e^{-m y e^{q / m}}\right\} .
\end{aligned}
$$

Lemma 4. For all $(x, y) \in R_{1}^{2}$ and $n, m \in N$, we have

$$
\begin{aligned}
& P_{n, m}\left(\left(t_{1}-x\right)^{2} ; x, y\right) \leq \frac{3(x+1)}{n} \\
& P_{n, m}\left(\left(t_{2}-y\right)^{2} ; x, y\right) \leq \frac{3(y+1)}{m} .
\end{aligned}
$$

Proof. By Lemma 1, we get

$$
\begin{aligned}
P_{n, m}\left(\left(t_{1}-x\right)^{2} ; x, y\right)= & (\operatorname{coth}(n x)-1) 2 x\left(\frac{1}{n} \operatorname{coth} 1-x\right)+\frac{x}{n} \operatorname{coth} n x \\
& +\frac{1}{n^{2}}(1+\operatorname{coth} 1) .
\end{aligned}
$$

Thus for $(x, y) \in R_{1}^{2}$, we can write

$$
P_{n, m}\left(\left(t_{1}-x\right)^{2} ; x, y\right) \leq \frac{x-1}{n}+\frac{2 x}{n}+\frac{3}{n^{2}} \leq \frac{3(x+1)}{n} .
$$

Similarly, we can easily obtain

$$
P_{n, m}\left(\left(t_{2}-y\right)^{2} ; x, y\right) \leq \frac{3(y+1)}{m} .
$$

Lemma 5. Let $p, q>0, r>p, s>q$ and let $n_{0}=n_{0}(p, r), m_{0}=m_{0}(q, s)$ be fixed natural numbers such that $n_{0}>p /(\ln r-\ln p)$ and $m_{0}>q /(\ln s-\ln q)$. Then there exist positive constants $C_{p, r}$ and $C_{q, s}$ depending only on $p, r$ and $q, s$ such that

$$
w_{r}(x) P_{n, m}\left(\left(t_{1}-x\right)^{2} e^{p t_{1}} ; x, y\right) \leq C_{p, r} \frac{\sinh \left(e^{p / n}\right)}{\sinh 1} \frac{x+2}{n}
$$




$$
w_{s}(y) P_{n, m}\left(\left(t_{2}-y\right)^{2} e^{q t_{2}} ; x, y\right) \leq C_{q, s} \frac{\sinh \left(e^{q / m}\right)}{\sinh 1} \frac{y+2}{m}
$$

for all $(x, y) \in R_{1}^{2}$ and $n \geq n_{0}, m \geq m_{0}$.

Proof. Firstly, for $m, n \in \mathbb{N}$, we consider the sequence of real numbers $\left(p_{n}\right)$ and $\left(q_{m}\right)$,

$$
\begin{aligned}
& p_{n}=n\left(e^{p / n}+1\right) \\
& q_{m}=m\left(e^{q / m}+1\right)
\end{aligned}
$$

which are decreasing and $\lim _{n \rightarrow \infty} p_{n}=p, \lim _{m \rightarrow \infty} q_{m}=q$. Thus

$$
\begin{aligned}
& p<p_{n}<p e^{p / n} \leq p e^{p} \\
& q<q_{m}<q e^{q / m} \leq q e^{q}
\end{aligned}
$$

Since $n_{0}>p /(\ln r-\ln p)$, we have $e^{p / n_{0}}<e^{\ln (r / p)}=r / p$ and $r>p e^{p / n_{0}}>p_{n_{0}}>$ $p_{n}$ for $n \geq n_{0}$. Also, because $m_{0}>q /(\ln s-\ln q)$, we get $e^{q / m_{0}}<e^{\ln (s / q)}=s / q$ and $s>q e^{q / m_{0}}>q_{m_{0}}>q_{m}$ for $m \geq m_{0}$.

Applying 2.1, we obtain

$$
\begin{aligned}
\sinh \left(n x e^{p / n}\right)(\sinh n x)^{-1} & \leq 2 e^{p_{n} x} \\
\cosh \left(n x e^{p / n}\right)(\sinh n x)^{-1} & \leq e^{p_{n} x} \\
x^{2}(\sinh n x)^{-1} & \leq \frac{x}{n}
\end{aligned}
$$

Also using 2.2, we get

$$
\begin{aligned}
\sinh \left(m y e^{q / m}\right)(\sinh m y)^{-1} & \leq 2 e^{q_{m} y} \\
\cosh \left(m y e^{q / m}\right)(\sinh m y)^{-1} & \leq e^{q_{m} y} \\
y^{2}(\sinh m y)^{-1} & \leq \frac{y}{m} .
\end{aligned}
$$

By writing the last inequalities in Lemma 2, we get respectively

$$
\begin{aligned}
& P_{n, m}\left(e^{p t_{1}} ; x, y\right) \leq \frac{\sinh \left(e^{p / n}\right)}{\sinh 1} 2 e^{p_{n} x} \\
& P_{n, m}\left(e^{q t_{2}} ; x, y\right) \leq \frac{\sinh \left(e^{q / m}\right)}{\sinh 1} 2 e^{q_{m} y}
\end{aligned}
$$


and

$$
\begin{aligned}
\left\|P_{n, m}\left(e^{p t_{1}} ; x, y\right)\right\|_{r} & \leq \sup \frac{\sinh \left(e^{p / n}\right)}{\sinh 1} 2 e^{\left(p_{n}-r\right) x} \\
\left\|P_{n, m}\left(e^{q t_{2}} ; x, y\right)\right\|_{s} & \leq \sup \frac{\sinh \left(e^{q / m}\right)}{\sinh 1} 2 e^{\left(q_{m}-s\right) y} .
\end{aligned}
$$

Taking into account Lemma 3 and 2.5, we obtain

$$
\begin{aligned}
& P_{n, m}\left(\left(t_{1}-x\right)^{2} e^{p t_{1}} ; x, y\right) \\
& \leq \frac{1}{\sinh 1}\left\{2 x^{2} e^{p_{n} x} \frac{p_{n}^{2}}{n^{2}} \sinh \left(e^{p / n}\right)+\frac{2 x}{n} \sinh \left(e^{p / n}\right) e^{\frac{p}{n}-n x e^{p / n}}\right. \\
&+\frac{2}{n^{2}} e^{p_{n} x} e^{2 p / n} \sinh \left(e^{p / n}\right)+\frac{2}{n^{2}} e^{p_{n} x} e^{p / n} \cosh \left(e^{p / n}\right) \\
&-\frac{4 x}{n} e^{p_{n} x} e^{p / n} \cosh \left(e^{p / n}\right)+\frac{2 x}{n} e^{p_{n} x} e^{2 p / n} \cosh \left(e^{p / n}\right) \\
&\left.+\frac{x}{n} e^{p_{n} x} e^{p / n} \sinh \left(e^{p / n}\right)\right\} \\
& \leq \frac{\sinh \left(e^{p / n}\right)}{\sinh 1}\left\{2 x^{2} \frac{p_{n}^{2}}{n^{2}} e^{p_{n} x}+\frac{2 x}{n} e^{p / n}+\frac{2}{n^{2}} e^{p_{n} x} e^{2 p / n}\right. \\
&+\frac{2}{n^{2}} e^{p_{n} x} e^{p / n} \operatorname{coth}\left(e^{p / n}\right)+\frac{2 x}{n} e^{p_{n} x} e^{2 p / n} \operatorname{coth}\left(e^{p / n}\right) \\
&\left.+\frac{x}{n} e^{p_{n} x} e^{p / n}\right\}
\end{aligned}
$$

Since $\operatorname{coth}\left(e^{p / n}\right) \leq \operatorname{coth} 1<2$ and $e^{p / n}<e^{p}$, we can write

$$
\begin{aligned}
P_{n, m}\left(\left(t_{1}-x\right)^{2} e^{p t_{1}} ; x, y\right) & \leq \frac{\sinh \left(e^{p / n}\right)}{\sinh 1}\left\{\frac{2 x^{2}}{n^{2}} p_{n}^{2} e^{p_{n} x}+\frac{2 x}{n} e^{p}+\frac{2}{n^{2}} e^{p_{n} x} e^{2 p}\right. \\
& \left.+\frac{4}{n^{2}} e^{p_{n} x} e^{p}+\frac{4 x}{n} e^{p_{n} x} e^{2 p}+\frac{x}{n} e^{p_{n} x} e^{p}\right\} .
\end{aligned}
$$

Say $w_{r}(x)=e^{-r x}$. Thus, we get

$$
\begin{aligned}
w_{r}(x) P_{n, m}\left(\left(t_{1}-x\right)^{2} e^{p t_{1}} ; x, y\right) \leq & \frac{\sinh \left(e^{p / n}\right)}{\sinh 1}\left\{\frac{2 x}{n} e^{\left(p_{n}-r\right) x}\left(\frac{x}{n} p_{n}^{2}+\frac{e^{p}}{2}+2 e^{2 p}\right)\right. \\
& \left.+\frac{2 x}{n} e^{p-r x}+\frac{2}{n^{2}} e^{\left(p_{n}-r\right) x}\left(e^{2 p}+2 e^{p}\right)\right\} .
\end{aligned}
$$


Now, by using 2.1 and inequalities

it follows

$$
\frac{x}{n} p_{n}^{2}<\frac{x}{n} p^{2} e^{2 p / n}<x p^{2} e^{2 p},
$$

$$
\begin{aligned}
& w_{r}(x) P_{n, m}\left(\left(t_{1}-x\right)^{2} e^{p t_{1}} ; x, y\right) \\
& \leq \frac{\sinh \left(e^{p / n}\right)}{\sinh 1}\left\{\frac{2 x}{n} e^{\left(p_{n}-r\right) x}\left(x p^{2} e^{2 p}+\frac{e^{p}}{2}+2 e^{2 p}\right)\right. \\
& \left.+\frac{2 x}{n} e^{p}+\frac{2}{n^{2}} e^{\left(p_{n}-r\right) x}\left(e^{2 p}+2 e^{p}\right)\right\} .
\end{aligned}
$$

Also, we have $r-p_{n} \geq r-p_{n_{0}}>0$ and $x e^{-\left(r-p_{n}\right) x} \leq x e^{-\left(r-p_{n_{0}}\right) x} \leq 1 /\left(r-p_{n_{0}}\right)$ for $n \geq n_{0}$. Applying $e^{\left(p_{n}-r\right) x}<1$, we obtain

$$
\begin{aligned}
& w_{r}(x) P_{n, m}\left(\left(t_{1}-x\right)^{2} e^{p t_{1}} ; x, y\right) \\
& \leq \frac{\sinh \left(e^{p / n}\right)}{\sinh 1}\left\{\frac{2}{n} \frac{1}{r-p_{n_{0}}}\left(x p^{2} e^{2 p}+2 e^{2 p}+\frac{e^{p}}{2}\right)\right. \\
&\left.+\frac{2 x}{n} e^{p}+\frac{2}{n^{2}}\left(e^{2 p}+2 e^{p}\right)\right\} \\
& \leq \frac{\sinh \left(e^{p / n}\right)}{\sinh 1}\left\{\frac{2 x}{n} \frac{e^{2 p}}{r-p_{n_{0}}} p^{2}+\frac{2}{n} \frac{1}{r-p_{n_{0}}}\left(2 e^{2 p}+\frac{e^{p}}{2}\right)\right. \\
&\left.+\frac{2 x}{n} e^{p}+\frac{2}{n^{2}}\left(e^{2 p}+2 e^{p}\right)\right\} \\
& \leq C_{p, r} \frac{\sinh \left(e^{p / n}\right)}{\sinh 1} \frac{x+2}{n} .
\end{aligned}
$$

Similarly as above, applying 2.6 to Lemma 3 , by simple calculations we easily obtain the required inequality

$$
w_{s}(y) P_{n, m}\left(\left(t_{2}-y\right)^{2} e^{q t_{2}} ; x, y\right) \leq C_{q, s} \frac{\sinh \left(e^{q / m}\right)}{\sinh 1} \frac{y+2}{m} .
$$

Lemma 6. If $p, q>0, r>p, s>q$ and $n_{0}=n_{0}(p, r), m_{0}=m_{0}(q, s)$ be fixed natural numbers such that $n_{0}>p /(\ln r-\ln p), m_{0}>q /(\ln s-\ln q)$ and if $f \in$ $C_{p, q}$, then we have

$$
\left\|P_{n, m}(f ; x, y)\right\|_{r, s} \leq 2\|f\|_{p, q} \frac{\sinh \left(e^{p}\right) \sinh \left(e^{q}\right)}{(\sinh 1)^{2}} .
$$


Proof. By (1), we can write

$$
\begin{aligned}
& e^{-r x} e^{-s y}\left|P_{n, m}(f ; x, y)\right| \\
& =\frac{e^{-r x} e^{-s y}}{(\sinh 1)^{2} \sinh (n x) \sinh (m y)} \mid \sum_{k=0}^{\infty} \sum_{j=0}^{\infty} p_{2 k}(n x) p_{2 j}(m y) e^{-\frac{2 k p}{n}} e^{-\frac{2 j q}{m}} \\
& \cdot f\left(\frac{2 k}{n}, \frac{2 j}{m}\right) e^{\frac{2 k p}{n}} e^{\frac{2 j q}{m}} \mid .
\end{aligned}
$$

Since $\|f\|_{p, q}=\sup _{(x, y) \in R_{1}^{2}} e^{-p x} e^{-q y}|f(x, y)|$, it follows that

$$
\begin{aligned}
& e^{-r x} e^{-s y}\left|P_{n, m}(f ; x, y)\right| \\
& \leq \frac{e^{-r x} e^{-s y}}{(\sinh 1)^{2} \sinh (n x) \sinh (m y)}\|f\|_{p, q} \sum_{k=0}^{\infty} \sum_{j=0}^{\infty} p_{2 k}(n x) p_{2 j}(m y) e^{\frac{2 k p}{n}} e^{\frac{2 j q}{m}} \\
& =e^{-r x} e^{-s y}\|f\|_{p, q} P_{n, m}\left(e^{p t_{1}} ; x, y\right) P_{n, m}\left(e^{q t_{2}} ; x, y\right) \\
& =e^{-r x} e^{-s y}\|f\|_{p, q} \frac{\sinh \left(e^{p / n}\right) \sinh \left(n x e^{p / n}\right)}{\sinh 1 \sinh (n x)} \frac{\sinh \left(e^{q / m}\right) \sinh \left(m y e^{q / m}\right)}{\sinh 1 \sinh (m y)} .
\end{aligned}
$$

Using the notations in Lemma 5, the inequalities (2.5) and (2.6), we obtain

$$
\begin{aligned}
e^{-r x} e^{-s y}\left|P_{n, m}(f ; x, y)\right| & \leq\|f\|_{p, q} e^{-r x} e^{-s y} \frac{\sinh \left(e^{p / n}\right)}{\sinh 1} 2 e^{p_{n} x} \frac{\sinh \left(e^{q / m}\right)}{\sinh 1} 2 e^{q_{m} y} \\
& =4\|f\|_{p, q} e^{-x\left(r-p_{n}\right)} e^{-y\left(s-q_{m}\right)} \frac{\sinh \left(e^{p / n}\right) \sinh \left(e^{q / m}\right)}{(\sinh 1)^{2}} \\
& \leq 4\|f\|_{p, q} \frac{\sinh \left(e^{p}\right) \sinh \left(e^{q}\right)}{(\sinh 1)^{2}}
\end{aligned}
$$

\section{Approximation by $\mathbf{P}_{n, m}$ Operators}

In this section, we give theorems on the degree of approximation of functions of two variables by these operators.

Theorem 1. Let $p, q>0, r>p, s>q$ and $n_{0}=n_{0}(p, r), m_{0}=m_{0}(q, s)$ be fixed natural numbers such that $n_{0}>p /(\ln r-\ln p)$ and $m_{0}>q /(\ln s-\ln q)$. If $f \in$ $C_{p, q}^{1}$, where $C_{p, q}^{1}=\left\{f \in C_{p, q}: f_{x}, f_{y} \in C_{p, q}\right\}$, then there exists a positive constant $M_{p, q, r, s}$ depending only on $p, q, r, s$ such that 


$$
\begin{gathered}
w_{r, s}(x, y)\left|P_{n, m}(f ; x, y)-f(x, y)\right| \\
\leq M_{p, q, r, s}\left\{\left\|\frac{\partial f}{\partial x}\right\|_{p, q} \sqrt{\frac{x+2}{n}}+\left\|\frac{\partial f}{\partial y}\right\|_{p, q} \sqrt{\frac{y+2}{m}}\right\}
\end{gathered}
$$

Proof. Let $(x, y)$ be a fixed point in $R_{1}^{2}$. For $f \in C_{p, q}^{1}$ and $\left(t_{1}, t_{2}\right) \in R_{1}^{2}$ we have

$$
f\left(t_{1}, t_{2}\right)-f(x, y)=\int_{x}^{t_{1}} \frac{\partial f}{\partial u}\left(u, t_{2}\right) d u+\int_{y}^{t_{2}} \frac{\partial f}{\partial v}(x, v) d v .
$$

Using $P_{n, m}(1 ; x, y)=1$, it results that

$$
\begin{gathered}
P_{n, m}\left(f\left(t_{1}, t_{2}\right) ; x, y\right)-f(x, y) \\
=P_{n, m}\left(\int_{x}^{t_{1}} \frac{\partial f}{\partial u}\left(u, t_{2}\right) d u ; x, y\right)+P_{n, m}\left(\int_{y}^{t_{2}} \frac{\partial f}{\partial v}(x, v) d v ; x, y\right) .
\end{gathered}
$$

For $r>p, s>q$ and $m, n \in \mathbb{N}$, we have

$$
\begin{aligned}
& w_{r, s}(x, y)\left|P_{n, m}\left(f\left(t_{1}, t_{2}\right) ; x, y\right)-f(x, y)\right| \\
& \leq w_{r, s}(x, y) P_{n, m}\left(\left|\int_{x}^{t_{1}} \frac{\partial f}{\partial u}\left(u, t_{2}\right) d u\right| ; x, y\right) \\
& \quad+w_{r, s}(x, y) P_{n, m}\left(\left|\int_{y}^{t_{2}} \frac{\partial f}{\partial v}(x, v) d v\right| ; x, y\right) .
\end{aligned}
$$

By using the following inequalities

$$
\begin{aligned}
\left|\int_{x}^{t_{1}} \frac{\partial f}{\partial u}\left(u, t_{2}\right) d u\right| & \leq\left\|\frac{\partial f}{\partial x}\right\|_{p, q}\left|\int_{x}^{t_{1}} \frac{1}{w_{p, q}\left(u, t_{2}\right)} d u\right| \\
& \leq\left\|\frac{\partial f}{\partial x}\right\|_{p, q} \frac{1}{w_{q}\left(t_{2}\right)}\left(\frac{1}{w_{p}\left(t_{1}\right)}+\frac{1}{w_{p}(x)}\right)\left|t_{1}-x\right|, \\
\left|\int_{y}^{t_{2}} \frac{\partial f}{\partial v}(x, v) d v\right| & \leq\left\|\frac{\partial f}{\partial y}\right\|_{p, q}\left|\int_{y}^{t_{2}} \frac{1}{w_{p, q}(x, v)} d v\right| \\
& \leq\left\|\frac{\partial f}{\partial y}\right\|_{p, q} \frac{1}{w_{p}(x)}\left(\frac{1}{w_{q}\left(t_{2}\right)}+\frac{1}{w_{q}(y)}\right)\left|t_{2}-y\right|
\end{aligned}
$$


and Hölder inequality, we can write

$$
\begin{aligned}
& w_{r, s}(x, y) P_{n, m}\left(\left|\int_{x}^{t_{1}} \frac{\partial f}{\partial u}\left(u, t_{2}\right) d u\right| ; x, y\right) \\
& \leq\left\|\frac{\partial f}{\partial x}\right\|_{p, q} w_{r, s}(x, y) P_{n, m}\left(\frac{1}{w_{q}\left(t_{2}\right)} ; x, y\right)\left\{P_{n, m}\left(\frac{\left|t_{1}-x\right|}{w_{p}\left(t_{1}\right)} ; x, y\right)\right. \\
& \left.+\frac{1}{w_{p}(x)} P_{n, m}\left(\left|t_{1}-x\right| ; x, y\right)\right\} \\
& \leq\left\|\frac{\partial f}{\partial x}\right\|_{p, q}\left\{\left[w_{r}(x) P_{n, m}\left(\left(t_{1}-x\right)^{2} e^{p t_{1}} ; x, y\right)\right]^{1 / 2}\left[w_{r}(x) P_{n, m}\left(e^{p t_{1}} ; x, y\right)\right]^{1 / 2}\right. \\
& \left.+e^{(p-r) x}\left[P_{n, m}\left(\left(t_{1}-x\right)^{2} ; x, y\right)\right]^{1 / 2}\right\} w_{s}(y) P_{n, m}\left(e^{q t_{2}} ; x, y\right), \\
& \quad w_{r, s}(x, y) P_{n, m}\left(\left|\int_{y}^{t_{2}} \frac{\partial f}{\partial v}(x, v) d v\right| ; x, y\right) \\
& \leq\left\|\frac{\partial f}{\partial y}\right\|_{p, q} \frac{w_{r}(x)}{w_{p}(x)} P_{n, m}(1 ; x, y) w_{s}(y)\left\{P_{n, m}\left(\frac{\left|t_{2}-y\right|}{w_{q}\left(t_{2}\right)} ; x, y\right)\right. \\
& \left.+\frac{1}{w_{q}(y)} P_{n, m}\left(\left|t_{2}-y\right| ; x, y\right)\right\} \\
& \leq\left\|\frac{\partial f}{\partial y}\right\|_{p, q}\left\{\left[w_{s}(y) P_{n, m}\left(\left(t_{2}-y\right)^{2} e^{q t_{2}} ; x, y\right)\right]^{1 / 2}\right. \\
& \left.\cdot\left[w_{s}(y) P_{n, m}\left(e^{q t_{2}} ; x, y\right)\right]^{1 / 2}+\left[P_{n, m}\left(\left(t_{2}-y\right)^{2} ; x, y\right)\right]^{1 / 2}\right\} .
\end{aligned}
$$

Applying the inequalities 2.5 and 2.6 to Lemma 2, we obtain

$$
\begin{aligned}
w_{r}(x) P_{n, m}\left(e^{p t_{1}} ; x, y\right) & \leq e^{-r x} \frac{\sinh \left(e^{p / n}\right)}{\sinh 1} 2 e^{p_{n} x} \\
& =2 \frac{\sinh \left(e^{p / n}\right)}{\sinh 1} e^{-x\left(r-p_{n}\right)} \\
& \leq 2 \frac{\sinh \left(e^{p / n}\right)}{\sinh 1}
\end{aligned}
$$

and

$$
w_{s}(y) P_{n, m}\left(e^{q t_{2}} ; x, y\right) \leq e^{-s y} \frac{\sinh \left(e^{q / m}\right)}{\sinh 1} 2 e^{q_{m} y}
$$




$$
\begin{aligned}
& =2 \frac{\sinh \left(e^{q / m}\right)}{\sinh 1} e^{-y\left(s-q_{m}\right)} \\
& \leq 2 \frac{\sinh \left(e^{q / m}\right)}{\sinh 1} .
\end{aligned}
$$

By these inequalities, Lemma 5 and Lemma 4, we get to

$$
\begin{aligned}
& w_{r, s}(x, y) P_{n, m}\left(\left|\int_{x}^{t_{1}} \frac{\partial f}{\partial u}\left(u, t_{2}\right) d u\right| ; x, y\right) \\
& \leq 2\left\|\frac{\partial f}{\partial x}\right\|_{p, q} \frac{\sinh \left(e^{p / n}\right) \sinh \left(e^{q / m}\right)}{(\sinh 1)^{2}} \sqrt{2 C_{p, r} \frac{x+2}{n}} \\
& +2\left\|\frac{\partial f}{\partial x}\right\|_{p, q} \frac{\sinh \left(e^{q / m}\right)}{\sinh 1} e^{-x(r-p)} \sqrt{\frac{3(x+1)}{n}} \\
& \leq\left\|\frac{\partial f}{\partial x}\right\|_{p, q} M_{p, r} \sqrt{\frac{x+2}{n}},
\end{aligned}
$$

and

$$
\begin{aligned}
& w_{r, s}(x, y) P_{n, m}\left(\left|\int_{y}^{t_{2}} \frac{\partial f}{\partial v}(x, v) d v\right| ; x, y\right) \\
& \leq\left\|\frac{\partial f}{\partial y}\right\|_{p, q} \frac{\sinh \left(e^{q / m}\right)}{\sinh 1} \sqrt{2 C_{q, s} \frac{y+2}{m}}+\left\|\frac{\partial f}{\partial y}\right\|_{p, q} \sqrt{\frac{3(y+1)}{m}} \\
& \leq\left\|\frac{\partial f}{\partial y}\right\|_{p, q} M_{q, s} \sqrt{\frac{y+2}{m}}
\end{aligned}
$$

for all $m \geq m_{0}$ and $n \geq n_{0}$. This proves the theorem.

Theorem 2. Suppose that $f \in C_{p, q}$ and $p, r, q, s, n_{0}, m_{0}$ satisfy the conditions of Theorem 1. Then there exists positive constant $M^{*}=M_{p, q, r, s}$ depending only on $p, q, r, s$ such that

$$
w_{r, s}(x, y)\left|P_{n, m}(f ; x, y)-f(x, y)\right| \leq M^{*} \omega\left(f, C_{p, q} ;\left(\frac{x+2}{n}\right)^{1 / 2},\left(\frac{y+2}{m}\right)^{1 / 2}\right)
$$

for all $(x, y) \in R_{1}^{2}$ and $m \geq m_{0}, n \geq n_{0}$. 
Proof. Similarly as in [5], we consider the Steklov means for $f \in C_{p, q}$

$$
f_{h, \delta}(x, y)=\frac{1}{h \delta} \int_{0}^{h} \int_{0}^{\delta} f(x+u, y+v) d u d v \quad, \quad h, \delta>0,(x, y) \in R_{1}^{2} .
$$

We have

$$
\begin{aligned}
f_{h, \delta}(x, y)-f(x, y) & =\frac{1}{h \delta} \int_{0}^{h} \int_{0}^{\delta} \Delta_{u, v} f(x, y) d u d v, \\
\frac{\partial f_{h, \delta}}{\partial x}(x, y) & =\frac{1}{h \delta} \int_{0}^{\delta}(f(x+h, y+v)-f(x, y+v)) d v, \\
\frac{\partial f_{h, \delta}}{\partial y}(x, y) & =\frac{1}{h \delta} \int_{0}^{h}(f(x+u, y+\delta)-f(x+u, y)) d u,
\end{aligned}
$$

which implies $f_{h, \delta} \in C_{p, q}^{1}(h, \delta>0)$ and

$$
\begin{gathered}
\left\|f_{h, \delta}-f\right\|_{p, q} \leq w\left(f, C_{p, q} ; h, \delta\right), \\
\left\|\frac{\partial f_{h, \delta}}{\partial x}\right\|_{p, q} \leq \sup _{(x, y) \in R_{1}^{2}} w_{p, q}(x, y) \frac{1}{h \delta} \int_{0}^{\delta}\left(\left|\Delta_{h, v} f(x, y)\right|+\left|\Delta_{0, v} f(x, y)\right|\right) d v \\
\leq \frac{2}{h} w\left(f, C_{p, q} ; h, \delta\right)
\end{gathered}
$$

and

$$
\left\|\frac{\partial f_{h, \delta}}{\partial y}\right\|_{p, q} \leq \frac{2}{\delta} w\left(f, C_{p, q} ; h, \delta\right)
$$

for $h, \delta>0$.

For every fixed $(x, y) \in R_{1}^{2}, r>p, s>q$ and $n, m \in \mathbb{N}, h, \delta>0$ we have

$$
\begin{aligned}
& w_{r, s}(x, y)\left|P_{n, m}(f ; x, y)-f(x, y)\right| \\
& \leq w_{r, s}(x, y)\left\{\left|P_{n, m}\left(f-f_{h, \delta} ; x, y\right)\right|\right. \\
& \left.\quad+\left|P_{n, m}\left(f_{h, \delta} ; x, y\right)-f_{h, \delta}(x, y)\right|+\left|f_{h, \delta}(x, y)-f(x, y)\right|\right\} .
\end{aligned}
$$

By Lemma 5, one obtains

$$
w_{r, s}(x, y)\left|P_{n, m}\left(f-f_{h, \delta} ; x, y\right)\right| \leq 4 w\left(f, C_{p, q} ; h, \delta\right)
$$

for all $m \geq m_{0}$ and $n \geq n_{0}$. Since Theorem 1 , we can write

$$
w_{r, s}(x, y)\left|P_{n, m}\left(f_{h, \delta} ; x, y\right)-f_{h, \delta}(x, y)\right|
$$




$$
\leq M_{p, q, r, s} w\left(f, C_{p, q} ; h, \delta\right)\left\{\frac{1}{h} \sqrt{\frac{x+2}{n}}+\frac{1}{\delta} \sqrt{\frac{y+2}{m}}\right\}
$$

for $m \geq m_{0}, n \geq n_{0}$. Therefore

$$
\begin{aligned}
& w_{r, s}(x, y)\left|P_{n, m}(f ; x, y)-f(x, y)\right| \\
& \leq 4 w\left(f, C_{p, q} ; h, \delta\right)+M_{p, q, r, s} w\left(f, C_{p, q} ; h, \delta\right)\left\{\frac{1}{h} \sqrt{\frac{x+2}{n}}+\frac{1}{\delta} \sqrt{\frac{y+2}{m}}\right\} \\
& \quad+w_{r, s}(x, y)\left|f_{h, \delta}(x, y)-f(x, y)\right| \\
& \leq w\left(f, C_{p, q} ; h, \delta\right)\left(5+M_{p, q, r, s}\left\{\frac{1}{h} \sqrt{\frac{x+2}{n}}+\frac{1}{\delta} \sqrt{\frac{y+2}{m}}\right\}\right)
\end{aligned}
$$

for all $h, \delta>0$ and $m \geq m_{0}, n \geq n_{0}$. Setting $h=\sqrt{\frac{x+2}{n}}, \delta=\sqrt{\frac{y+2}{m}}$ we obtain the desired result.

Corollary 1. If $f \in C_{p, q}$, then for all $(x, y) \in R_{1}^{2}$

$$
\lim _{m, n \rightarrow \infty} P_{n, m}(f ; x, y)=f(x, y) .
$$

Also, the convergence is uniform on every rectangle $1 \leq x \leq a, 1 \leq y \leq b$.

\section{REFERENCES}

[1] A. Ciupa, "Approximation by a generalized Szasz type operator," J. Comput. Anal. Appl., vol. 5, no. 4, pp. 413-424, 2003.

[2] J. Favard, "Sur les multiplicateurs d'interpolation," J. Math. Pures Appl., vol. 9, no. 23, pp. 219247, 1944.

[3] Z. Finta, N. K. Govil, and V. Gupta, "Some results on modified Szász-Mirakjan operators," J. Math. Anal. Appl., vol. 327, no. 2, pp. 1284-1296, 2007.

[4] A. Jakimovski and D. Leviatan, "Generalized Szasz operators for the approximation in the finite interval." Mathematica, Cluj, vol. 11, pp. 97-103, 1969.

[5] M. Leśniewicz and L. Rempulska, "Approximation by some operators of the Szasz-Mirakjan type in exponential weight spaces," Glas. Mat., III. Ser., vol. 32, no. 1, pp. 57-69, 1997.

[6] R. N. Mohapatra and Z. Walczak, "Remarks on a class of Szász-Mirakyan type operators," East J. Approx., vol. 15, no. 2, pp. 197-206, 2009.

[7] C. Mortici, "An extension of the Szász-Mirakjan operators," An. Ştiinţ. Univ. "Ovidius" Constanţa, Ser. Mat., vol. 17, no. 1, pp. 137-144, 2009.

[8] L. Rempulska and S. Graczyk, "On certain class of Szász-Mirakyan operators in exponential weight spaces," Int. J. Pure Appl. Math., vol. 60, no. 3, pp. 259-267, 2010.

[9] Z. Walczak, "On the convergence of the modified Szasz-Mirakyan operators," Yokohama Math. J., vol. 51, no. 1, pp. 11-18, 2004. 
Authors' addresses

\section{Nursel Çetin}

Ankara University, Faculty of Science, Department of Mathematics, Tandogan 06100 Ankara, Turkey

E-mail address: ncetineankara.edu.tr

\section{Sevilay Kirci Serenbay}

Başkent University, Department of Mathematics Education, 06530 Ankara, Turkey

E-mail address: kirci@baskent.edu.tr

\section{Çiğdem Atakut}

Ankara University, Faculty of Science, Department of Mathematics, Tandogan 06100 Ankara, Turkey

E-mail address: atakut@science.ankara.edu.tr 